\title{
HISTOLOGICAL INVESTIGATIONS ON THE INFLUENCE OF DEXAMETHASONE ON RAT TEMPOROMANDIBULAR JOINT INDUCED ARTHRITIS
}

\author{
Mohamed Ibrahim Halawa*, Abdelhafez Elhussiny Suliman*, Glal Abo elyazeed Nasr ${ }^{* * *}$
}

\begin{abstract}
In this study, 30 female Sprague-Dawley rats were used and were divided into three groups including: group (I): the control group involved 10 female rats, group (II): involved 10 female rats subjected to osteoarthritis induction without treatment, group (III): involved 10 female rats treated with dexamethasone after osteoarthritis induction. Experimental procedures in osteoarthritic animals a single dose of Formalin $(1.5 \%)$ or its vehicle $(0.9 \% \mathrm{NaCl})$ was unilaterally injected in the TMJ $(30 \mu \mathrm{L})$ with a Hamilton syringe connected to a 30_gauge needle introduced into the TMJ at the moment of injection. After 1week post osteoarthritis induction, the treatments with Dexamethasone will be administered for 4 weeks as follow: Group III: the experimental animals were treated with a single dose $(50 \mu \mathrm{L})$ of $0.12 \mathrm{mg}$ dexamethasone via intra-articular route as prescribed previously. After osteoarthritis induction, in group (II), fusion between disc and condyle, condensation of the cells in the fibrous layer, approachment of bone marrow spaces to the cartilagenous layer, defaseiculation of central part of articular disc, Chondrocytes appear condensed, degeneration of numerous cells, variable in size and pyknotic nuclei, erosion in the condylar fibrous and cartilaginous layer, synovial membrane became extensively proliferated to develope synovial villi into the temporodiscal space. After treatment with dexamethasone, in group (III), the histopathological features became significantly lowered after the treatment with dexamethasone when compared with the control group, the inflammatory reactions had been inhibited, although slight hyperplasia of the synovial membrane cells, the condylar cartilage appeared of variable thickness while the chondrocytes showed marked proliferation, variable in size and irregular distribution.
\end{abstract}

\section{INTRODUCTION}

Pain in the temporomandibular joint (TMJ) region is a major symptom associated with temporomandibular disorders (TMDs) and is recognized as one of the most difficult types of pain to treat. ${ }^{(1)}$ It was demonstrated that the experimentally induced nociception is higher in female than in male rats ${ }^{(2,3)}$. These findings are in accordance with the higher prevalence and severity of TMDs in women than in men ${ }^{(4)}$.

The inflammation of TMJ is one of the TMDs that may cause reduced mandibular and craniofacial growth. It may also cause unstable occlusion and disturbed the TMJ and masticatory function which may lead to asymmetric loading on joints and muscles, orofacial symptoms, and a compromised aesthetic appearance ${ }^{(5)}$. The management goals for patients with TMDs include decreased pain, reduced adverse loading, restoration of the function, and resumption of normal daily activities ${ }^{(6)}$.

The pharmacologic intervention with corticosteroids and non-steroidal anti-inflammatory drugs (NSAIDs) were tried successfully in the treatment of TMDs ${ }^{(7)}$. The intra-articular therapy with corticosteroids is a well-established treatment for TMJ arthritis, The joint injection with corticosteroid produced good anti-inflammatory results because the

\footnotetext{
* Demonstrator of Oral Histology, Oral Biology Department, Faculty of Dental Medicine Boys, Al-Azhar University, Cairo ** Professor of Oral Histology, Oral Biology Department, Faculty of Dental Medicine Boys, Al-Azhar University, Cairo *** Lecturer of Oral Histology, Oral Biology Department, Faculty of Dental Medicine Boys, Al-Azhar University, Cairo
} 
drug efficacy was maximized and directed to the affected area and all the adverse effects from the systemic administration were avoided ${ }^{(8,9,10)}$. The TMJ is functionally important for chewing, speaking, swallowing and movement of the mandible ${ }^{(11)}$. TMJ-OA may be unilateral or bilateral and its strong preference for women may be due to estrogen receptor alpha polymorphism and may be associated with increased pain susceptibility in female TMJ-OA patients ${ }^{(12,13)}$.

Mechanical and biological events including overloading, bruxism, and unilateral chewing, as well as genetic factors and internal derangement are causative factors in the development of TMJOA which causes not only focal degeneration of the joint cartilage, but also osseous erosion, sclerosis, flattening, and osteophyte formation at the joint margins ${ }^{(13,14)}$. The increased risk factors induce a homeostatic imbalance between the anabolic (synthesis) and catabolic (degeneration) conditions in the articular cartilage of the TMJ. Subsequently, a homeostatic imbalance induces an upregulation of the pro-inflammatory cytokines, such as interleukin (IL)-1 $\beta$, tumor necrosis factor- $\alpha$, and IL- 6 and catabolic growth factors in the synovial fluids of the $\mathrm{TMJ}^{(15)}$.

These events, subsequently, cause that the degradation of the cartilage outweighs cartilage formation, resulting in the development of grooves in the joint surface, cracks or ulcers, and loss of cartilage, resulting in exposure of the subchondral bone, osteophyte formation, and subchondral cysts ${ }^{(16)}$.

In general, the natural course of TMJ osteoarthritis is favorable ${ }^{(17)}$ and can be divided into three slow progressive phases, with periods of remission and cartilage regeneration ${ }^{(18)}$. The initial stage where there is evolution of the condition is termed early phase. This may take on average 2.5-4 years. Clinically it is associated with clicking sounds and intermittent locking. The intermediate phase, associated with TMJ destruction, lasts on average 6 months to one year and clinically the patient may undergo spontaneous joint pain at rest or with function, limitation in opening, and grating sounds. The late phase is the stage at which there is no degenerative activity, and the joints are said to be stable or in the "burnout phase". It lasts about 6 months, and it will eventually stabilize with time and therefore, if invasive procedures can be postponed with medical management, patients will ultimately benefit from it. There is absence of joint pain, absence or presence of limitation, absence or presence of grating sounds. The entire process from initiation to the final burnout phase takes approximately 5.5 years $^{(19)}$.

The most common clinical signs and symptoms include pain, restriction in joint function, and joint sounds. Pain is usually dull aching and may have occasional sharp component on movement. Pain is prevalent in initial phases due to the presence of synovitis ${ }^{(18)}$. It may be associated with joint stiffness, limitation in mouth opening, increasing sensitivity to cold and damp and may be relieved with rest, and NSAIDs. Patients usually have morning stiffness for more than $30 \mathrm{~min}$, joint crepitus, joint sounds and absence of joint warmth. Patients in advanced stages may be exhibit facial skeletal remodeling, with chin deviation towards the affected side, unstable or fluctuating malocclusion with occlusal discrepancies ${ }^{(20)}$. Occlusal changes like skeletal anterior open bite, reduced overbite and increased overjet may be associated with osteoarthritic TMJ (21). In addition, internal derangements may coexist in the same joint in approximately one-third of cases $^{(22)}$.

The following were the most frequent morphological changes observed: flattening of the anterior surface of the condyle; followed by erosions and irregularities of the joint surfaces; flattening of the articular surface of the temporal eminence, subchondral cysts, osteophytes; and idiopathic condyle resorption ${ }^{(25)}$. 
Treatment of TMJ osteoarthritis should be directed at suppressing the active inflammatory process, preserving function, preventing further deformity and relieving pain. Management is largely symptomatic. Studies have shown that nonsurgical treatment can successfully be used to treat patients with osteoarthritis ${ }^{(21)}$. Treatment includes physical therapy, pulsed electrical stimulation, pharmacological, topical ointments, supplements, steroid injections, HA injections, acupuncture. ${ }^{(19)}$

Intra-articular injection of local anesthetics and corticosteroids has also been suggested following failure to oral medications. Methyl-prednisolone and triamcinolone acetonide have been used. A systematic review concluded that lavage of the TMJ may be slightly more effective than nonsurgical treatment for pain reduction. However, this difference is not likely to be clinically relevant ${ }^{(26)}$.

Chemically induced models mostly involve the injection of a toxic or inflammatory compound directly into the joint, this model can be used to study the effects of drugs on the inflammation or pain caused by these substances. Papain, sodium monoiodoacetate, quinolone, and collagenase are some of the chemicals employed to induce OA in animals, they eliminate the need for surgery and avoid possible infection issues in some animals. Their ease of induction and reproducibility are advantageous in designing short term studies ${ }^{(27) .}$

\section{MATERIALS AND METHODS}

In this study, 30 female Sprague-Dawley rats were used and were divided into three groups including: group (I): the control group involved 10 female rats, group (II): involved 10 female rats subjected to osteoarthritis induction without treatment, group (III): involved 10 female rats treated with dexamethasone after osteoarthritis induction.

Experimental procedures in osteoarthritic animals a single dose of Formalin $(1.5 \%)$ or its vehicle $(0.9 \% \mathrm{NaCl})$ was unilaterally injected in the
TMJ $(30 \mu \mathrm{L})$ with a Hamilton syringe connected to a 30_gauge needle introduced into the TMJ at the moment of injection. Formalin was prepared from commercially (Sigma Chemicals) available stock formalin (an aqueous solution of $37 \%$ of formaldehyde) further diluted in $0.9 \% \mathrm{NaCl}$ to a concentration of $1.5 \%$.

\section{The experimental animals were injected through the following protocol:}

After shaving the area over the TMJ, the joint felt $5-10 \mathrm{~mm}$ posterior to the lateral canthus of the eye, while the mandible was manipulated to provide movement of the condyle for a further positive identification of the joint. The TMJ area was located by palpation over the zygomatic arch from anterior to posterior and from the interval between the medial aspect of the zygomatic arch and the skull. The zygomatic arch is attached posteriorly to the skull at the temporal bone. At this V-shaped notch, the glenoid fossa is inferior and posterior. Insulin syringe needle was then inserted from a postersuperior direction until the mandibular condyle was felt. In all experimental animals the right side were used for osteoarthritis induction, while the other side were left untreated.

After 1week post osteoarthritis induction, the treatments with Dexamethasone will be administered for 4 weeks as follow: Group III: the experimental animals were treated with a single dose $(50 \mu \mathrm{L})$ of $0.12 \mathrm{mg}$ dexamethasone (Dexamethasone produced by Amiriya Pharmaceutical Industries, Alexandria, Egypt. Under license of Weimer Pharma, GmbH, Rastatt, W.Germany). via intra-articular route as prescribed previously. After the end of the experiment period, the rats were anesthetized, sacrificed by euthanasia, and the skull was cut into two halves through sagittal plane, then each half was demineralized by EDTA, paraffin embedded and sagittal sections were prepared to demonstrate the TMJ. The tissue sections were micro technically processed for histological evaluations. 


\section{RESULTS}

The control group showed the histological component of the TMJ as condylar fibrous layer of glenoid fibrous layer, Condylar cartilage layer and glenoid cartilage layer. Temporodiscal space and condylodiscal space. Subarticular bone and bone marrow. The fibrous layer, covering the condylar head consisted of numerous fibroblasts scattered through dense parallelly arranged avascular layers of collagen fibers (Fig.1).

The histological changes in TMJ induced arthritis by formalin injection showed Erosion in the condylar fibrous and cartilaginous layer and severe degenerative changes in the TMJ, dehiscence, splitting and fibrosis of the articular disc, lack of dense collagen bundles and disruption of collagen bundles in certain areas of fibrocartilaginous layer, lack of cartilaginous layer, there are area of resorption within the cartilaginous zone and cartilage bone interface, osteophytes and resorption of the surface of condyle with widening of marrow spaces and necrosis of marrow tissue (fig.2) the eroded area may be replaced by fatty tissue, the erosion may extend to sub articular bone, the cartilaginous layer does not exist and approachment of bone marrow

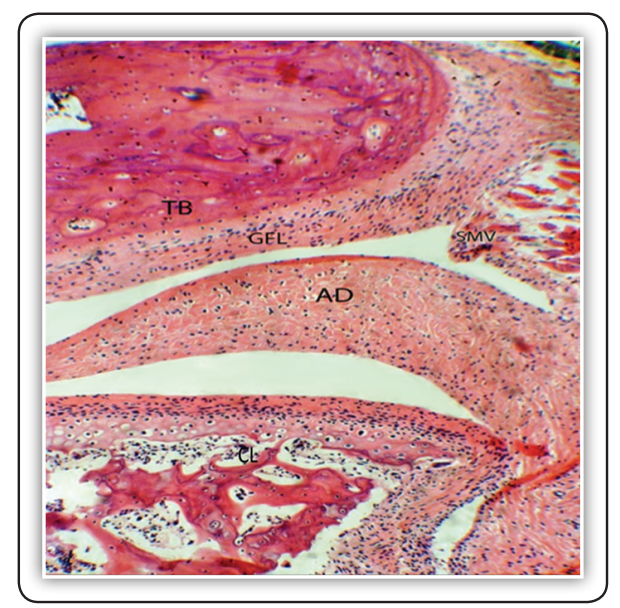

FIG (1) Photomicrograph from sagittal section of female rat TMJ of control group (GI) showing Synovial membrane villi (SMV) Glenoid fibrous layer (GFL) Temporal bone (TB) Articular disc (AD) condylar bone (CL): (H \& E stain, orig. mag. X100). spaces near to fibrous layer were seen. A fusion of the central part of articular disc to condylar fibrous layer, thinning of condylar cartilage layer and increasing of the thickness of condylar fibrous layer (fig.3).

The TMJ of dexamethasone treated rats showed a relative improvement was noted in all parts of the temporomandibular joint in the covering fibrous layer, cartilaginous zone of mandibular condyle and the articular disc. The condylar bone of mandibular condyle showed normal bone marrow spaces (fig.4), while the condylar cartilage showing marked extensive proliferation of the chondrocytes with marked increasing in the thickness of the condylar cartilaginous layer than normal case. The zone of cartilage/ bone interface is well demarcated and makes the transitional layer between the articular cartilage and the underlying trabecular bone. There are areas of resorption within the cartilaginous layer and cartilage/ bone interface. (fig.5). Fusion of the central part of the articular disc with the fibrous layer of the roof of glenoid fossa, widening in the condylodiscal space and narrowing in the temporodiscal space (fig.3)

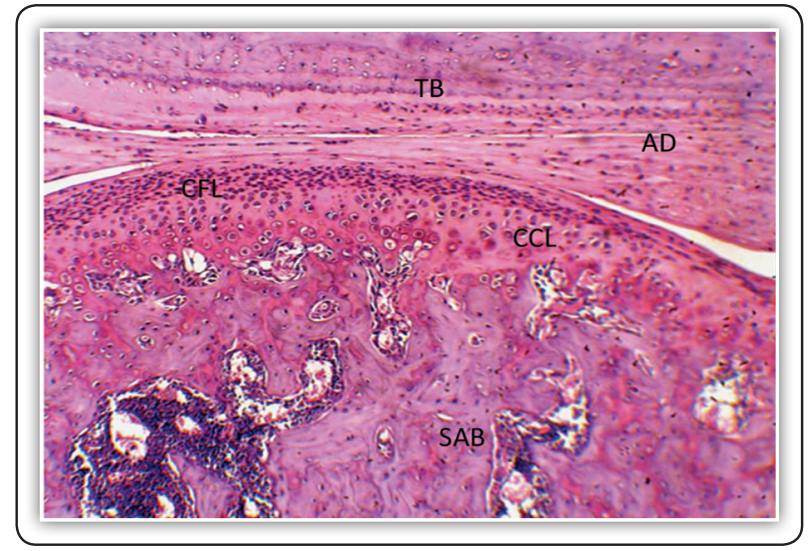

FIG (2) Photomicrograph from sagittal section in female rat TMJ of the osteoarthritic group (GII) showing fusion of the central part of articular disc (AD) to condylar fibrous layer (CFL) with irregular thickness of condylar cartilage layer (CCL). (H\& E stain, orig. mag. X100). Temporal bone (TB)

Sub articular bone (SAB) 


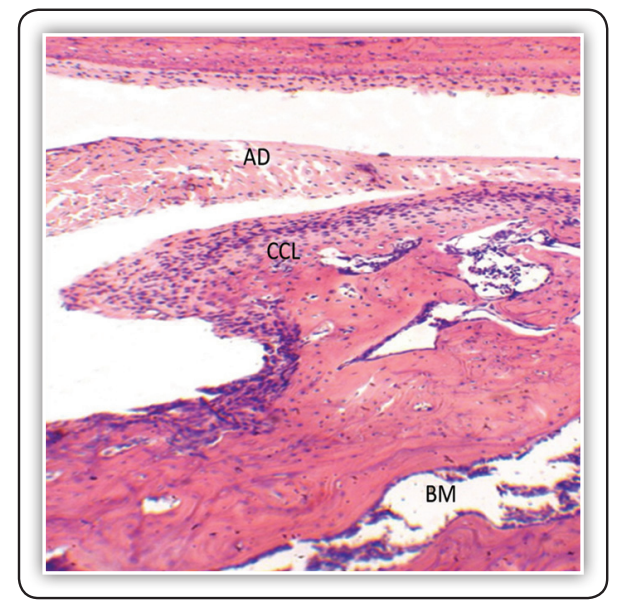

FIG (3) Photomicrograph from sagittal section in female rat TMJ of the osteoarthritic group (GII) showing severe degenerative changes in the TMJ, dehiscence, splitting and fibrosis of the articular disc (AD), lack of dense collagen bundles and disturption of collagen bundles in certain areas of fibrocartilaginous layer, lack of cartilaginous layer (CCL), there are area of resorption within the cartilaginous zone and cartilage bone interface, osteophytes and resorption of the surface of condyle with widening of marrow spaces (BM) and necrosis of marrow tissue. (H \& E stain, orig. mag. X100).

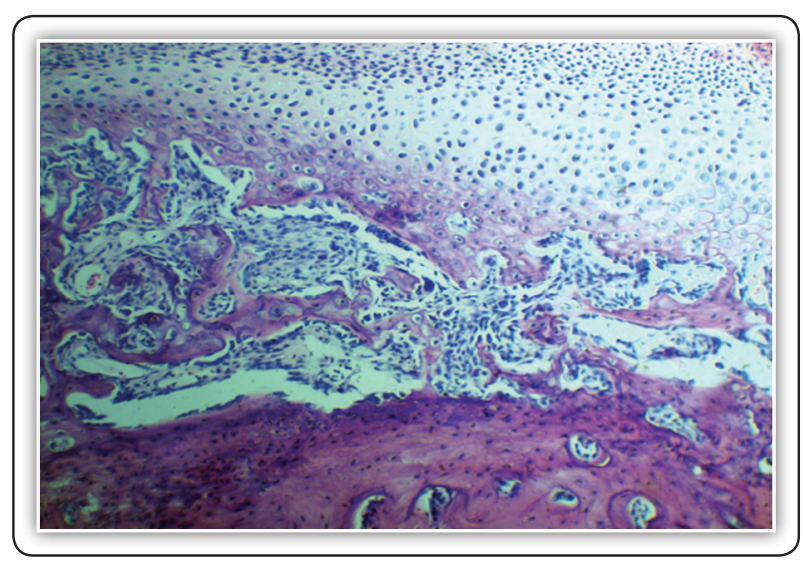

FIG (5) Photomicrograph from sagittal section of female rat at condylar head of dexamethasone treated group (GIII) showing marked extensive proliferation of the chondrocytes with marked increasing in the thickness of the condylar cartilaginous layer than normal case. The zone of cartilage/ bone interface is well demarcated and makes the transitional layer between the articular cartilage and the underlying trabecular bone. There are areas of resorption within the cartilaginous layer and cartilage/ bone interface. (H \& E stain, orig. mag. X100).

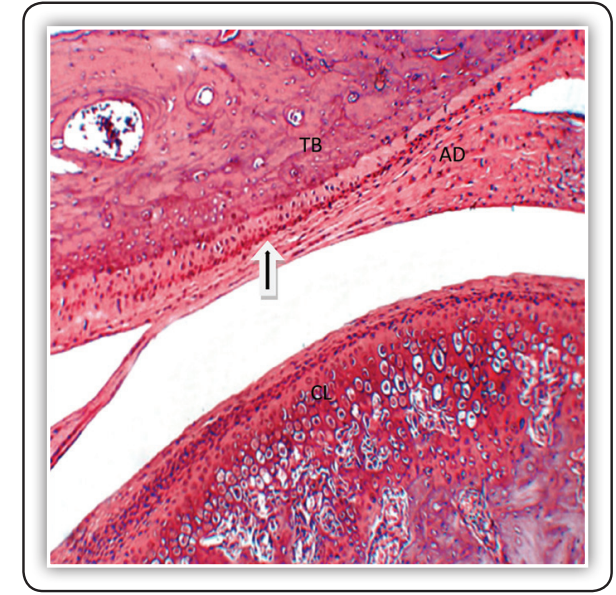

FIG (4) Photomicrograph from sagittal section of female rat TMJ of dexamethasone treated group (GIII) showing fusion of the articular disc (AD) with the fibrous layer of the roof of glenoid fossa (arrow), increasing in the thickness of the condylar cartilaginous layer and normal histological structure of temporal bone. ( $\mathrm{H} \& \mathrm{E}$ stain, orig. mag. X100).

\section{DISCUSSION}

TMDs are defined as a wide group of pathological conditions involving pain and dysfunction in the masticatory system. It is a multifactorial condition that has different signs and symptoms in the joints and its structural components ${ }^{(28)}$. Several studies presumed that the inflammatory disorders are considered as the inducing mechanism underlying many of the clinical symptoms referred to as arthritis. Its manifestations include group of variable inflammatory disorders in which the degenerative joints changes are the outstanding feature ${ }^{(28)}$. The present study has been designed to investigate the effects of dexamethasone and simvastatin as a treatment for TMJ arthritis of female rats. Though the rats have no articular eminence ${ }^{(29)}$. however, the rats were used as experimental animals due to close morphological and histological similarity of the articular structures of the rats to those of humans.

\section{Effect of osteoarthritis induction on TMJ}

The present work has proved that the TMJ of osteo-arthrised rats show variable microscopical 
changes in response to intra-articular formalin injection. The most interesting histological finding was the erosion in the condylar fibrous and cartilaginous layer and severe degenerative changes in the TMJ, dehiscence, splitting and fibrosis of the articular disc, lack of dense collagen bundles and disturption of collagen bundles in certain areas of fibrocartilaginous layer, lack of cartilaginous layer, there are area of resorption within the cartilaginous zone and cartilage bone interface, osteophytes and resorption of the surface of condyle with widening of marrow spaces and necrosis of marrow tissue, fusion of the central part of the articular disc to the fibrous and cartilagenous layers of the mandibular condyle. Also an irregular thickness of the condylar cartilagenous layers were seen associated with the condensation of condrocytes. However, the chondrocytes of the condylar cartilage were atrophied as evidenced with their variable reduction in size and pyknotic nuclei. Synovial membrane also showed hyperplasia and extensively proliferation of synovial villi into temporodiscal space. The histochemical results demonstrated that the amount of neutral mucosubstances were more in the TMJ of control group. These findings were similar to those revealed by many previous investigators Cömert Kiliç S, Güngörmüş M. 2016 and Mark RE. 2004 proved that TMJ-OA causes focal degeneration of joint cartilage, erosion, flattening and osteophyte formation ${ }^{(13,14)}$ were in the same line with this study also agree with these observations. Also El-Hakim IE, Abdel-Hamid IS, Bader A. 2005 revealed the same observations on synovial membrane as a result for mechanical osteoarthritic induction ${ }^{(30)}$. Goulart AC, et al. 2005 showed intense inflammatory infiltration, hyperplasia of synovial membrane with villous changes and thickening of condylar articular surface with atrophy of fibrocartilage ${ }^{(31)}$. These investigations were compatible with our study.

\section{Significance of dexamethasone}

In the present study, the dexamethasone drug has been selected due to its anti-inflammatory effect, this drug has been evaluated by many investigators and was tested and compared, it was found that the dexamethasone was the potent drug in regards to treatment of the inflammation and is used to treat certain forms of arthritis; skin, blood, kidney, eye, thyroid, and intestinal disorders. In respect to the dexamethasone dose used in the present study (a single dose $(50 \mu \mathrm{L})$ of $0.12 \mathrm{mg}$ dexamethasone via intra-articular route) which is well within the reported range. The mortality rate in the present study revealed that animals about $(10 \%)$ were died at different intervals during experimental period and were replaced with other animals. This may indicate that this animal models are compatible to this drug. Hubner K.D, et al. 2014 proved that dexamethasone inhibits inflammation and cartilage damage in models of post traumatic osteoarthritis ${ }^{(32)}$, Also El-Hakim IE, Abdel-Hamid IS, Bader A. 2005 proved the effect of dexamethasone on TMJ synovitis as it decreased the synovial inflammation. The previous studies concluded that the results support the concern that the dexamethasone might protect the TMJ component as synovial membrane and cartilaginous layer from the deleterious effect of osteoarthritis. However, the present results did not support the finding reported previously by other investigators ${ }^{(33,34)}$. These researchers found that glucocorticoid might be chondrotoxic in vitro.

\section{The effect of dexamethasone injection on TMJ}

In the present study the dexamethasone was used one time after one week from the induction of osteoarthritis. The results demonstrated that it has relatively protect the TMJ component from the injurious effect of osteoarthritis, The histological results demonstrated a relative improvement in all parts of the TMJ in the covering fibrous layer, cartilaginous zone of mandibular condyle and the articular disc, the condylar cartilage appeared of variable thickness while the chondrocytes showed marked proliferation. These relative improvements in deleterious alternations may be due to the corrective effect of dexamethasone. These results 
are in agreements with the findings of previous studies who studied the anti-inflammatory effect of dexamethasone on arthritis. Numerous studies have investigated the efficacy of intra-articular injections of corticosteroids in the management of OA symptoms. In a review Ringdahl and Pandit ${ }^{(35)}$ found that corticosteroid injections reduced pain in the short-term compared to placebo.

\section{CONCLUSIONS}

The present findings, as well as, the previous investigations have proved that harmful histological changes on TMJ structure occur with intra articular injection with formalin. The therapeutic effect of dexamethasone is clear as it inhibited the inflammatory reactions.

\section{REFERENCES}

1. Torres-Chaves KE, Sanfins JM, Clemente-Napimoga JT, Pelegrini-Da-Silva A, et al. Effect of gonadal steroid hormones on formalin-induced temporomandibular joint inflammation. Pain. 2012; 16: 204-216.

2. Bereiter DA, Benetti AP. Amino acid release at the spinomedullary junction after inflammation of TMJ region in male and female rats. Pain. 2006; 126:175-183.

3. Carins BE, Sim Y, Bereiter DA, Sessle BJ, et al. Influence of sex on reflex jaw muscle activity evoked from the rat temporomandibular joint. Brain Res. 2002; 957:338-344.

4. Fischer L, Torres-Chaves KE, Clemente-Napimoga JT, Jorge D, Arsati F, et al. The influence of sex and ovarian hormones on temporomandibular joint nociception in rats. J Pain. 2008; 9:630-638.

5. Stoustrup P, Kristensen KD, Verna C, Kuseler A, et al Intra-articular steroid injection for temporomandibular joint arthritis in juvenile idiopathic arthritis: A systematic review on efficacy and safety. Seminars in Arthritis and Rheumatism.2013; 43:63-70.

6. Okeson JP. Orofacial Pain. Guidlines for assessment, diagnosis, and management. London: Quintessence Publishing Co; Inc.1996:141-158.

7. Syrop SB. Non-surgical management of temporomandibulr disorders. In: Peterson LJ, Indresano A, Marciani RD; Principles of Oral and Maxillofacial Surgery. Vol 3. Philadelphia: Lippincott-Ravan publishers 1997:1905-1931.
8. Neidel J. Intra-articular steroid therapy for inflammatory rheumatoid diseases in children and adolescents. Orthopade $2002 ; 31: 1175-1178$.

9. Kopp S, Akerman S, Nilner M. Short-term effects of intraarticular sodium hyaluronate, glucocorticoid, and saline injections on rheumatoid arthritis of the temporomandibular joint. J Craniomand Disord 1991; 5:231-238.

10. Shore NA. Temporomandibular joint Dysfunction and Occlusal Equilibration. Philadelphia: Lippincott Co.1976:213-233.

11. Rando C, Waldron T. TMJ osteoarthritis: a new approach to diagnosis. Am J Phys Anthropol. 2012 May; 148(1): 45-53.

12. Boyan BD, Tosi LL, Coutts RD, Enoka RM, et al. Addressing the gaps: sex differences in osteoarthritis of the knee. Biol Sex Differ. 2013 Feb 4; 4(1): 4.

13. Cömert Kiliç S, Güngörmüş M. Is arthrocentesis plus platelet-rich plasma superior to arthrocentesis plus hyaluronic acid for the treatment of temporomandibular joint osteoarthritis: a randomized clinical trial. J Oral Maxillofac Surg. 2016 Nov; 74(11): 2151-2158.

14. Mark RE. Platelet rich plasma: evidence to support its use. J Oral Mxillofac Surg. 2004; 62: 489-496.

15. Vernal R, Velásquez E, Gamonal J, Garcia-Sanz JA, et al. Expression of proinflammatory cytokines in osteoarthritis of the temporomandibular joint. Arch Oral Biol. 2008 Oct; 53(10): 910-105.

16. Da Costa FA, Souza DC, de Araújo GM, Gurgel CV,et al. Does TGF- $\beta$ play a role in degenerative temporomandibular joint diseases? A systematic review. Cranio. $2017 \mathrm{Jul}$; 35(4): 228-232.

17. Manfredini D, Favero L, Gregorini G, Cocilovo F, et al. Natural course of temporomandibular disorders with low pain-related impairment: a 2-to-3-year follow-up study. J Oral Rehabil 2013 Jun; 40(6): 436-42.

18. Wang XD, Kou XX, He DQ, Zeng MM, et al. Progression of cartilage degradation, bone resorption and pain in rat temporomandibular joint osteoarthritis induced by injection of iodoacetate. PLoS One 2012; 7(9): 36-45.

19. Kalladka M, Quek S, Heir G, Eliav E, et al. Temporomandibular joint osteoarthritis: diagnosis and long-term conservative management: a topic review. J Indian Prosthodont Soc. 2014 Mar; 14(1): 6-15.

20. Chen YJ, Shih TT, Wang JS, Wang HY, et al. Magnetic resonance images of the temporomandibular joints of patients with acquired open bite. Oral Surg Oral Med Oral Pathol Oral Radiol Endod 2005; 99(6): 734-742. 
21. Tanaka E, Detamore MS, Mercuri LG. Degenerative disorders of the temporomandibular joint: etiology, diagnosis, and treatment. J Dent Res 2008; 87(4): 296-307.

22. Schmitter M, Essig M, Seneadza V, Balke Z, et al. Prevalence of clinical and radiographic signs of osteoarthrosis of the temporomandibular joint in an older persons community. Dentomaxillofac Radiol 2010; 39: 231-234.

23. Nah KS. Condylar bony changes in patients with temporomandibular disorders: a CBCT study. Imaging Sci Dent. 2012 Dec; 42(4): 249-253.

24. Vos LM, Huddleston Slater JJ, Stegenga B. Lavage therapy versus nonsurgical therapy for the treatment of arthralgia of the temporomandibular joint: a systematic review of randomized controlled trials. J Orofac Pain 2013 Spring; 27(2): 171-179.

25. Kuyinu EL, Narayanan G, Nair LS, Laurencin CT. Animal models of osteoarthritis: classification, update, and measurement of outcomes. J Orthop Surg Res. 2016 Feb; 2: 11-19.

26. Mercuri LG. Osteoarthritis, osteoarthrosis, and idiopathic condylar resorption. Oral Maxillofac Surg Clin North Am 2008; 20(2):169-183.

27. Porto GG, Vasconcelos BC, Andrade ES, Silva-Junior VA.
Comparison between human and rat TMJ: anatomic and histopathologic features. Acta Cir. Bras 2010; 25:3.

28. El-Hakim IE, Abdel-Hamid IS, Bader A. Temporomandibular joint (TMJ) response to intra-articular dexamethasone injection following mechanical arthropathy: A histological study in rats. Int J Oral Maxillofac Surg 2005; 34: 305.

29. Goulart AC, Correia FA, Souza SC, Luz JG. Study of the inflammatory process induced by injection of carrageenan or formalin in the rat temporomandibular joint. Braz Oral Res 2005; 19(2): 99-105.

30. Huebner KD, Shrive NG, Frank CB. Dexamethasone Inhibits Inflammation and Cartilage Damage in a New Model of PostTraumatic Osteoarthritis. J Orthop Res 2014; 32:566-572.

31. Braun HJ, Wilcox-Fogel N, Kim HJ, et al. The effect of local anesthetic and corticosteroid combinations on chondrocyte viability. Knee Surg Sports Traumatol Arthrosc 2012; 20:1689-1695.

32. Dragoo JL, Danial CM, Braun HJ, et al. The chondrotoxicity of single-dose corticosteroids. Knee Surg Sports Traumatol Arthrosc 2012; 20:1809-1814.

33. Ringdahl E, Pandit S. Treatment of knee osteoarthritis. Am Fam Physician 2011; 83:1287-1292. 